\title{
Corrigendum
}

\section{Diagnostic radiography and adult acute myeloid leukemia: an interview and medical chart review study}

\section{JM Pogoda, PW Nichols, RK Ross, DO Stram, DC Thomas and S Preston-Martin}

British Journal of Cancer (2012) 1 07, 1204. doi:10.1038/bjc.2012.426 www.bjcancer.com

(c) 2012 Cancer Research UK

Correction to: British Journal of Cancer (2012) 104, 1482-1486, The full, correct Table 4 is now presented, below: doi:10.1038/bjc.2011.114

After publication, it was noted that the dose category labels contained in Table 4 of this paper were presented at magnitude 100 times higher than was correct.

Table 4 Risk analysis of total estimated bone marrow dose from diagnostic imaging procedures in the 2-10 years before diagnosis, adjusted for SES ${ }^{a}$

\begin{tabular}{|c|c|c|c|c|c|c|c|c|c|c|c|}
\hline \multirow[b]{2}{*}{ FAB group } & \multirow{2}{*}{$\begin{array}{l}\text { Total estimated } \\
\text { dose }(m G y)^{b}\end{array}$} & \multicolumn{5}{|c|}{ Data from medical records } & \multicolumn{5}{|c|}{ Data from interview } \\
\hline & & \# Ca & \# Co & OR & $(95 \% \mathrm{Cl})$ & $P$ trend $^{c}$ & \# Ca & \# Co & OR & $(95 \% \mathrm{Cl})$ & $P$ trend $^{c}$ \\
\hline All ${ }^{d}$ & $\begin{array}{c}\leqslant 10.00 \\
10.01-20.00 \\
>20.00\end{array}$ & $\begin{array}{r}234 \\
22 \\
23\end{array}$ & $\begin{array}{r}236 \\
30 \\
13\end{array}$ & $\begin{array}{l}1.0 \\
0.7 \\
1.6\end{array}$ & $\begin{array}{l}(0.4,1.3) \\
(0.8,3.2)\end{array}$ & 0.28 & $\begin{array}{r}295 \\
31 \\
18\end{array}$ & $\begin{array}{r}290 \\
46 \\
8\end{array}$ & $\begin{array}{l}1.0 \\
0.6 \\
2.5\end{array}$ & $\begin{array}{l}(0.4,1.0) \\
(1.0,6.0)\end{array}$ & 0.70 \\
\hline M2 & $\begin{array}{c}\leqslant 10.00 \\
10.01-20.00 \\
>20.00\end{array}$ & $\begin{array}{r}65 \\
6 \\
8\end{array}$ & $\begin{array}{r}66 \\
10 \\
3\end{array}$ & $\begin{array}{l}1.0 \\
0.7 \\
2.2\end{array}$ & $\begin{array}{l}(0.2,2.1) \\
(0.6,9.0)\end{array}$ & 0.54 & $\begin{array}{r}89 \\
7 \\
3\end{array}$ & $\begin{array}{r}84 \\
10 \\
5\end{array}$ & $\begin{array}{l}1.0 \\
0.6 \\
0.6\end{array}$ & $\begin{array}{l}(0.2,1.8) \\
(0.1,2.6)\end{array}$ & 0.46 \\
\hline M3 & $\begin{array}{c}\leqslant 10.00 \\
10.01-20.00 \\
>20.00\end{array}$ & $\begin{array}{r}22 \\
2 \\
3\end{array}$ & $\begin{array}{r}24 \\
3 \\
0\end{array}$ & $\begin{array}{l}1.0 \\
0.6 \\
-\end{array}$ & $(0.1,3.8)$ & 0.27 & $\begin{array}{r}27 \\
1 \\
5\end{array}$ & $\begin{array}{r}31 \\
2 \\
0\end{array}$ & $\begin{array}{l}1.0 \\
0.5 \\
-\end{array}$ & $\begin{array}{c}(0.0,5.5) \\
-\end{array}$ & 0.18 \\
\hline$M 5^{f}$ & $\begin{array}{c}\leqslant 10.00 \\
10.01-20.00 \\
>20.00\end{array}$ & $\begin{array}{r}17 \\
0 \\
2\end{array}$ & $\begin{array}{r}16 \\
2 \\
1\end{array}$ & $\begin{array}{l}1.0 \\
0.0 \\
1.9\end{array}$ & $\begin{array}{l}(0.0,-) \\
(0.1,53.1)\end{array}$ & 0.63 & $\begin{array}{r}18 \\
1 \\
2\end{array}$ & $\begin{array}{r}19 \\
2 \\
0\end{array}$ & $\begin{array}{l}1.0 \\
0.6 \\
-\end{array}$ & $(0.1,7.7)$ & 0.96 \\
\hline
\end{tabular}

Abbreviations: $\mathrm{Ca}=$ cases; $\mathrm{Cl}=$ confidence interval; $\mathrm{Co}=$ control; $\mathrm{FAB}=$ French-American-British; $\mathrm{OR}=$ odds ratio; $\mathrm{SES}=$ socioeconomic status. ${ }^{\mathrm{a}}$ Excludes pairs in which either the case or control had prior radiation therapy and/or chemotherapy for cancer. ${ }^{b}$ Dose is likely to be consistently underestimated (see text). ${ }^{\circ}$ Total dose as a continuous variable. ${ }^{d}$ Includes 5 I case-control pairs with unknown FAB subtype. ${ }^{e}$ Excludes cases with FAB subtype M4eo. Includes cases with FAB subtypes M5A and M5B. 